\title{
Ki-67 expression in pericoronal tissues of impacted mandibular third molars
}

\author{
Mahmut Sumer $^{a}$, Levent Yıldiz ${ }^{\mathrm{b}}$, A. Pinar Sumer*c, Samet Inal ${ }^{\mathrm{d}}$ \\ ${ }^{a}$ Department of Oral and Maxillofacial Surgery, Faculty of Dentistry, Ondokuz Mayis University, Samsun, Turkey \\ ${ }^{b}$ Department of Pathology, Faculty of Medicine, Ondokuz Mayis University, Samsun, Turkey \\ ${ }^{c}$ Department of Dentomaxillofacial Radiology, Faculty of Dentistry, Ondokuz Mayis University, Samsun, Turkey \\ ${ }^{d}$ Oral and Maxillofacial Surgery, Private Center, Antalya, Turkey
}

\begin{tabular}{l}
\hline ARTICLE INFO \\
\hline $\begin{array}{l}\text { Article History } \\
\text { Received }\end{array}$ \\
\begin{tabular}{ll} 
Accepted & $24 / 11 / 2011$ \\
& $11 / 12 / 2011$ \\
\hline
\end{tabular}
\end{tabular}

\section{* Correspondence to}
A. Pinar Sumer
Ondokuz Mayıs Üniversitesi,
Dişhekimliği Fakültesi,
A ğı $1 z$ Diş ve Çene Radyolojisi
Anabilim Dal1,
Kurupelit, Samsun, Türkiye
e-mail: psumer1970@yahoo.com

\section{Keywords:}

Impacted tooth

Third molar

Dental follicle

Dentigerous cyst

Ki-67

Cell neoplastic transformation

\begin{abstract}
The study was performed to histologically and immunohistochemically evaluate soft tissue pathologic changes in pericoronal epithelial tissues of impacted mandibular third molars that did not exhibit pathologic pericoronal radiolucency and to clarify the role of Ki-67 expression for separation between healthy follicle and dentigerous cyst. Fifty pericoronal tissues associated with completely impacted mandibular third molars were submitted for histologic examination by each of 2 pathologists after surgical tooth removal was performed. Biopsy samples were fixed in $10 \%$ buffered neutral formalin solution and embedded in paraffin blocks. Sections (4-6 $\mu \mathrm{m})$ were obtained from the paraffin block and to evaluate the expression of Ki-67 sections were immunostained anti-Ki-67 primary antibody and streptavidin-biotin complex system. The incidence of normal tissue of a dental follicle was $22 \%$, and the incidence of dentigerous cyst was $52 \%$, and the incidence of chronic nonspecific inflammatory tissue was $26 \%$. Ki-67 expression was positive in 19 of 26 lining epithelia in cystic specimens, in 4 of 13 chronic nonspecific inflammatory tissue specimens, and in 0 of 11 healthy follicular specimens. These findings suggest that the majority of cystic specimens demonstrated proliferative activity, therefore the incidence of dentigerous cyst associated with impacted third molar teeth is high and proliferative marker may be played a role in diagnosis of dentigerous cyst associated with radiographically normal third molar impactions.

J. Exp. Clin. Med., 2012; 29:9-12
\end{abstract}

\section{Introduction}

Removal of third molars is the most commonly performed procedure in oral and maxillofacial surgery. Debate concerning the indications for removal of impacted third molars has been ungoing in dentistry for many years. The decision to remove an impacted tooth is less challenging when signs and symptoms of pathologic changes are present. Although many pathological processes may present radiographically as pericoronal radiolucencies associated with impacted teeth, the most common is the dentigerous cyst. Pericoronal radiolucency less than $2.5 \mathrm{~mm}$ in diameter were suggested as nonpathologic (Eliason and Heimdahl, 1989). However, widely accepted criteria for differentiation between healthy dental follicle and dentigerous cyst do not exist, this remains an area of controversy (Curran et al., 2002).

Previous studies (Glosser and Campbell, 1999; Adelsperger et al., 2000; Rakprasitkul, 2001) have concluded that the incidence of soft tissue pathological conditions associated with impacted third molar teeth is higher than reported in radiographic studies alone. The study was performed to examine the follicular tissue associated with impacted mandibular third molars without pericoronal radiolucency and to clarify the role of Ki-67 expression for differentiation between healthy follicle and dentigerous cyst. To assess the incidence of cystic change, histologic examination was carried out and to assess the degree of epithelial proliferative activity, immunohistochemical study was performed.

\section{Material and methods}

Fifty patients who were referred to the oral surgery clinic for removal of completely impacted mandibular third molars were enrolled in this study. The patients selected for this study were healthy and without significant medical diseases or history of bleeding problems. Third molar teeth were removed for variety of reasons, especially orthodontics. Follicular spaces were measured from panoramic radiograph and 
impacted third molar with a follicular space less than $2.5 \mathrm{~mm}$ was submitted for evaluation. Teeth with pericoronal spaces larger than $2.4 \mathrm{~mm}$ and teeth without adequate follicular tissue for histologic evaluation were excluded.

Fifty impacted mandibular third molars were removed, and the pericoronal soft tissues of these were submitted for histopathologic examination by each of 2 pathologists. The pericoronal tissues were grouped into three categories according to the histological findings (Rakprasitkul, 2001):

1. Dental follicle

2. Dentigerous cyst

3. Chronic nonspecific inflammatory tissue

A diagnosis of dentigerous cyst was recorded when both pathologists diagnosed cystic change as evidenced by the presence of squamous metaplasia in the follicle lining (Fig. 1).

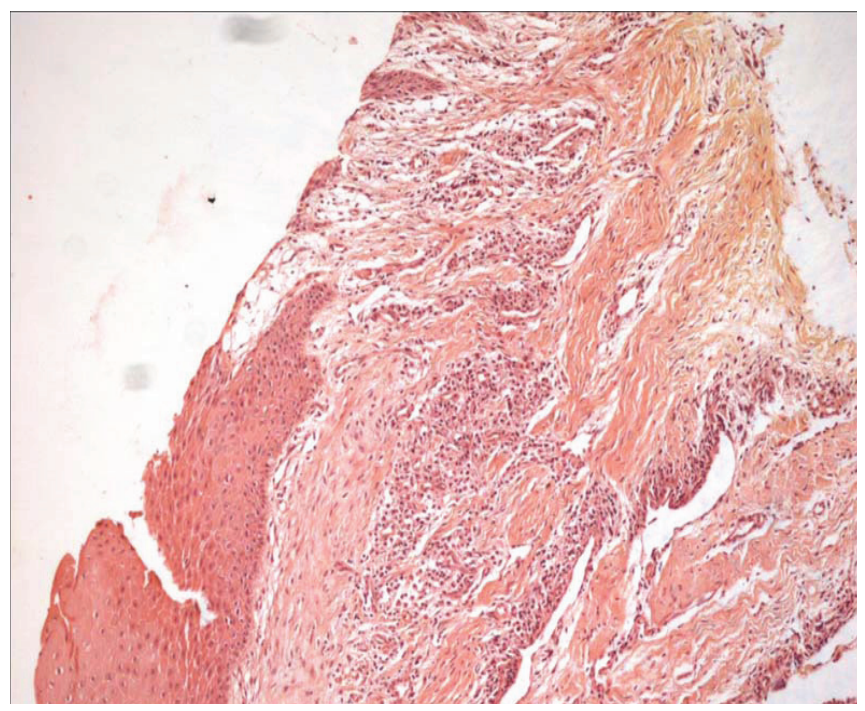

Fig. 1. Histologic appearance of a dentigerous cyst (x100, HE)

The criterion for dental follicle was the presence of remnants of enamel epithelium in the fibrous connective tissue layer, and the absence of an epithelial lining (Fig. 2).

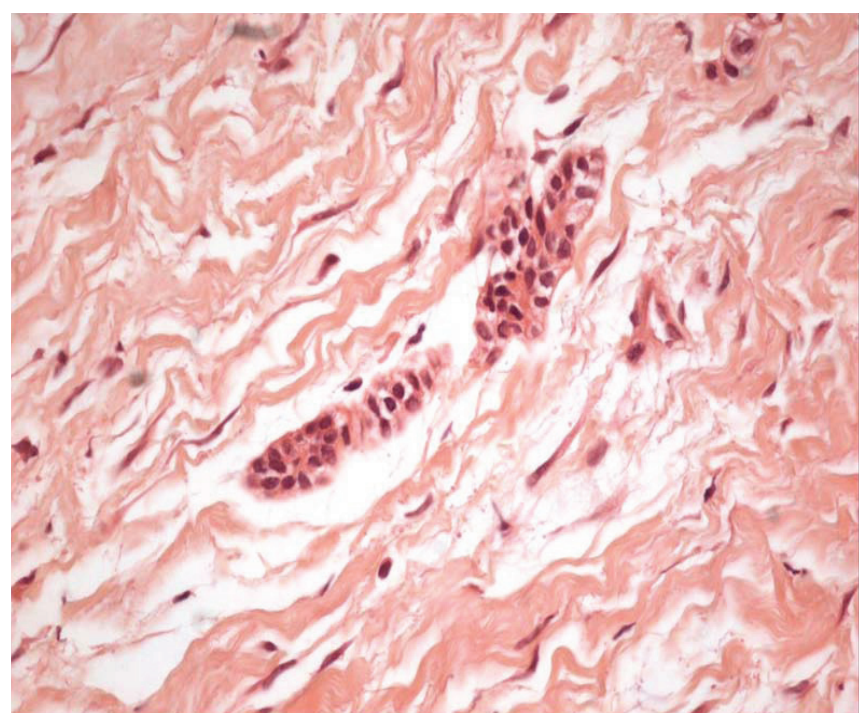

Fig. 2. Histologic appearance of a dental follicle (x200, HE)

Chronic nonspecific inflammatory tissue showed the presence of chronic granulation tissue and hyperplastic squamous epithelium in the soft tissues (Fig. 3).

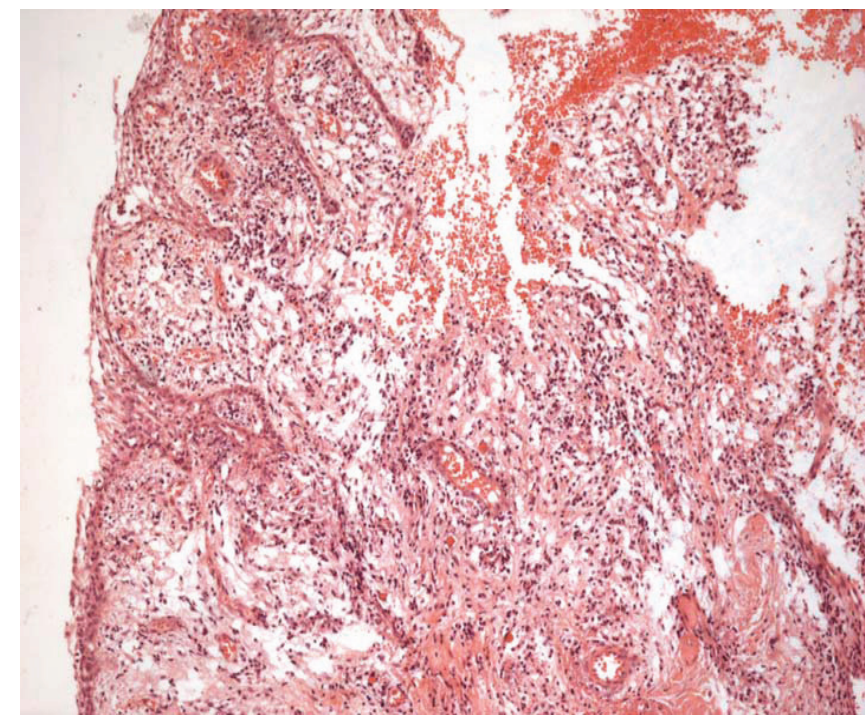

Fig. 3. Histologic appearance of chronic nonspecific inflammatory tissue (x200, HE)

Biopsy samples were fixed in 10\% buffered neutral formalin solution and embedded in paraffin. Sections $(4-6 \mu \mathrm{m})$ were obtained from the paraffin block and to evaluate the expression of Ki-67 sections were immunostained anti-Ki-67 primary antibody (monoclonal mouse, clone: MB67 Diagnostic biosystem, CA, USA) and streptavidin-biotin complex system (Diagnostic biosystem, CA, USA).

For Ki-67, the percentage of positive cell nuclei was calculated after analyzing about 1000 cells in five high-power fields of the lining epithelium and solid proliferations of remnant enamel epithelium.

\section{Results}

Patients' ages ranged between 16-36 years (mean, 23.44 \pm 5.32 ). Sixteen male and 34 female samples were acquired.

Of 50 specimens submitted for histologic examination, 11 specimens (22\%) were diagnosed as being dental follicle, 26 specimens $(52 \%)$ were diagnosed as dentigerous cysts, and 13 specimens $(26 \%)$ were diagnosed as chronic nonspecific inflammatory tissue.

The mean age for nonpathologic follicular tissue was $20.09 \pm 2.16$ years, the mean age for chronic nonspecific inflammatory tissue was $22.5 \pm 3.61$ years, and the mean age for dentigerous cysts was $25.3 \pm 6.2$ years. A statistically significant increase in the presence of squamous metaplasia of the follicular epithelium was present with advancing age.

Ki-67 expression was positive in 19 of 26 cystic specimens, in 4 of 13 chronic nonspecific inflammatory tissue specimens, and in 0 of 11 healthy follicular specimens. Ki-67 expression was negative in 7 of 26 cystic specimens, in 9 of 13 chronic nonspecific inflammatory tissue specimens, and in 11 of 11 healthy follicular specimens. This result showed that the majority of cystic specimens demonstrated proliferative activity (Fig. 4).

The percentage of Ki-67 positive nuclei was $8.99 \pm 9.54$ in dentigerous cysts and $1.87 \pm 4.14$ in chronic nonspecific inflammatory tissue. Statistically significant differences were found between the expression of Ki-67 in dentigerous cysts and chronic nonspecific inflammatory tissue $(p<0.05)$. 


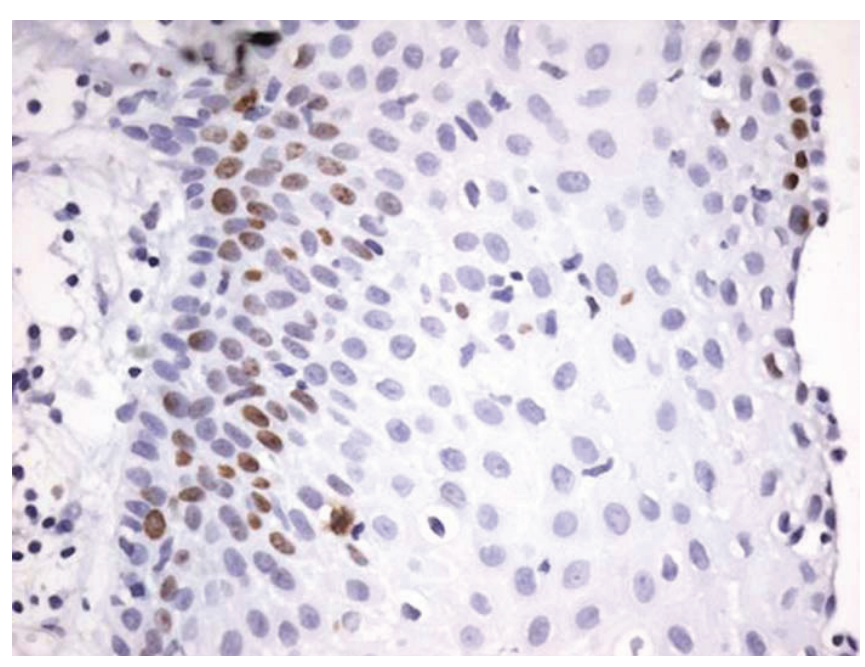

Fig. 4. A cystic specimen demonstrating Ki-67 positive nuclei indicative of epithelial proliferative activity (x400 streptavidin biotin labeling, DAB chromogen)

\section{Discussion}

The presence of disease associated with impacted third molar teeth is an indication for their removal; however removal for prevention of disease remains controversial. Odontogenic tissue surrounding impacted teeth has the potential to differentiate into a wide variety of tissue types, and the potential for cyst and tumor development exists (Gorlin, 1957). Leitner et al., (2007) reported a patient who was diagnosed with a lowgrade malignant fibrosarcoma of the dental follicle of an impacted third molar without clinical evidence of any follicular lesion. Prophylactic removal of impacted teeth was suggested even in cases which clinically and radiographically showed normal tissues (Brkic et al., 2010). The studies examined the cystic changes of radiographically normal follicles (Glosser and Campbell, 1999; Adelsperger et al., 2000; Rakprasitkul, 2001; Baykul et al., 2005) have reported higher incidence of cysts than expected. The results of this study showed that $52 \%$ of samples exhibited squamous metaplasia indistinguishable from the histological changes found in dentigerous cyst and $26 \%$ samples exhibited chronic nonspecific inflammatory tissue. This finding is consistent with previous reports (Rakprasitkul, 2001; Baykul et al., 2005), however shows a higher incidence that has been reported in other studies related with impacted third molars (Glosser and Campbell, 1999; Adel- sperger et al., 2000).

We found a correlation between the incidence of cystic change in follicular tissues and age. Previous authors have also reported an increasing incidence of dentigerous cyst formation as age increases (Glosser and Campbell, 1999; Adelsperger et al., 2000). There was a strong relationship between increasing age and pathologic changes (Curran et al., 2002).

Despite the high incidence of cystic changes, removal of third molars for the prevention of disease may not always necessary and it is also possible that these cystic areas do not progress to become larger destructive lesions (Glosser and Campbell, 1999). Additional histological criteria might be helpful for confirming both definition and diagnosis of dentigerous cyst. For this reason, in addition to histologic examination, we assess the degree of proliferative activity in radiographically normal follicles.

Detection of Ki-67 is considered a reliable marker of cellular proliferation (Gerdes et al., 1984). Ki-67 antigen expression has been observed in the nuclei of proliferating cells, and it can be a marker estimate the state of tissue growth (Piattelli et al., 2002). It has been reported that Ki-67 antigen expression increases in preneoplastic and neoplastic lesions of the oral mucosa and in all states of high cell turnover (Sano et al., 1998; Saito et al., 1999).

In this study, most tested pathologic specimens had demonstrable Ki-67 expression. Conversely, this was not demonstrated in any tested sample diagnosed as a healthy follicle. Ki-67 analysis demonstrated that a sizable number of these lesions were undergoing epithelial proliferation at the time of tooth extraction. Adelsperger et al. (2000) stained ten specimens with squamous metaplasia and 8 with healthy follicular epithelium with proliferating cell nuclear antigen (PCNA) to assess cellular proliferative activity and suggested that at least some specimens with squamous metaplasia might be actively proliferating, rather than quiescent lesions. These findings suggest that lack of radiographic appearance of disease is not a reliable indicator of the absence of disease and that the prevalence of soft tissue pathologic changes is higher than generally assumed from radiographic evaluation alone (Eliason and Heimdahl, 1989).

In conclusion under the conditions of this study, the incidence of dentigerous cyst associated with impacted third molar teeth is high and proliferative marker may be played a role in diagnosis of dentigerous cyst associated with radiographically normal third molar impactions.

\section{REFERENCES}

Adelsperger, J., Campbell, J.H., Coates, D.B., Summerlin, D.J., Tomich, C.E., 2000. Early soft tissue pathosis associated with impacted third molars without pericoronal radiolucency. Oral Surg. Oral Med. Oral Pathol. Oral Radiol. Endod. 89, 402-406.

Baykul, T., Saglam, A.A., Aydin, U., Basak, K., 2005. Incidence of cystic changes in radiographically normal impacted lower third molar follicles. Oral Surg. Oral Med. Oral Pathol. Oral Radiol. Endod. 99, 542-545.

Brkic, A., Mutlu, S., Berberoglu, H.K., Olgac, V., 2010. Pathological changes and immunoexpression of p63 gene in dental follicles of asymptomatic impacted lower third molars: An immunohistochemical study. J. Craniofac. Surg. 21, 854-857.

Curran, A.E., Damm, D.D., Drummond, J.F., 2002. Pathologically significant pericoronal lesions in adults: Histopathologic evaluation. J. Oral Maxillofac. Surg. 60, 613-617.

Eliason, S., Heimdahl, A., 1989. Pathologic changes related to long term impaction of third molars: A radiographic study. Int. J. Oral. Maxillofac. Surg. 18, 210-212.

Gerdes, J., Lemke, H., Baisch, H., Wacker, H.H., Schwab, U., Stein, H., 1984. Cell cycle analysis of a cell proliferation-associated human nuclear antigen defined by the monoclonal antibody Ki-67. J. Immunol. 133, 1710-1715.

Glosser, J.W., Campbell, J.H., 1999. Pathologic changes in soft tissues associated with radiographically normal third molar impactions. Br. J. Oral Maxillofac. Surg. 37, 259-260.

Gorlin, R.J., 1957. Potentialities of oral epithelium manifested by mandibular dentigerous cysts. Oral Surg. Oral Med. Oral Pathol. 10, 271. 
Leitner, C., Hoffmann, J., Kröber, S., Reinert, S., 2007. Low-grade malignant fibrosarcoma of the dental follicle of an unerupted third molar without clinical evidence of any follicular lesion. J. Craniofac. Surg. 35, 48-51.

Piattelli, A., Lezzi, G., Fioroni, M., Santinelli, A., Rubini, C., 2002. Ki-67 expression in dentigerous cysts, unicystic ameloblastomas, and ameloblastomas arising from dental cysts. J. Endod. 28, 55-58.

Rakprasitkul, S., 2001. Pathologic changes in the pericoronal tissues of unerupted third molars. Quintessence Int. 32, 633-638.

Saito, K., Mori, S., Tanda, N., Sakamoto, S., 1999. Expression of p53 protein and Ki-67 antigen in gingival hyperplasia induced by nifedipine and phenytoin. J. Periodontol. 70, 581-586.

Sano, K., Yoshida, S., Ninomiya, H., Ikeda, H., Ueno, K., Sekine, J., Iwamoto, H., Uehara, M., Inokuchi, T., 1998. Assessment of growth potential by MIB-1 immunohistochemistry in ameloblastic fibroma and related lesions of the jaws compared with ameloblastic fibrosarcoma. J. Oral Pathol. Med. 27, 59-63. 